\title{
DESIGN AND FABRICATION OF A 3-FIN SYMMETRIC RAY- INSPIRED SOFT ROBOT FOR UNDERWATER EXPLORATION
}

\author{
Muhammad Bilal Khan and Pruittikorn Smithmaitrie \\ Department of Mechanical Engineering, Faculty of Engineering, Prince of Songkla University, Hat Yai, \\ Songkhla, 90112, Thailand. \\ mbilalkhannu@gmail.com,pruittikorn.s@psu.ac.th
}

\begin{abstract}
Manta rays and stingrays have cartilaginous fins and disk-like bodies. Their unique shape facilitates efficient underwater locomotion. With inspiration of their natural evolution, the design and fabrication of a new ray inspired soft robot is presented. This soft robot is designed with a potential to perform underwater locomotion. An elastomeric soft body is developed by combining selective features of a manta ray and stingray. The concept is such that two of the side fins provide basic thrust and the third fin provides steering to the whole body. This three-fins concept is helpful for multi directional movement with minimal control adjustment. For actuation, each of three servo motors connects with a flexible cylindrical tendon. The actuation system is embedded within the elastomeric body of the ray robot. Without electronics installed, the body of the soft robot weights $36 \mathrm{~g}$ which is very light and suitable for locomotion by low power consumption. The soft fins are tested under controlled environments both outside and under water. The fin motion is tuned by investigating different flapping amplitudes and frequencies. By recording video of a fin motion, minor undulatory and major oscillatory motion components are observed across pectoral fins. The efficient pectoral fin gait is also identified by comparing the actuator power consumption for a fin flapping cycle. This work has shown that this new design of the soft ray robot can be an efficient swimming robot with design-and-material provided compliance. Further development would lead it to be a fully functional underwater exploratory robot.
\end{abstract}

Keywords: Biomimetic; rays; Soft robot; Underwater locomotion.

\section{Introduction}

In the recent decades, a number of bioinspired robots have been built with different levels of locomotion capabilities for many purposes and working in different environments [1].

These robots have different actuation approaches to perform locomotion by flying (in the air), walking or running (on the ground), and swimming (under water). By mimicking different animal locomotion principles in nature, the biomimicking robot locomotion efficiency is enhanced. This benefit supports the robots to do complicated tasks such as navigation to collect data from an environment, handling objects, and inspection of sites where human access may not be possible. Roughly $70 \%$ of the earth consists of water and it is mostly unexplored. This unexplored area could be reached by underwater vehicles [2]. A number of efforts have been made in order to design AUVs based on bioinspired approaches [2], [3].

Some of the robots are designed by mimicking aquatic animal morphology and movements, which is a fundamental part of behaviour-based robotics.
Among underwater locomotion systems, a number of works mimic morphology and locomotion behaviours of rays such as manta rays and stingrays [4]. Rays are the efficient under water swimmers [2], [5]-[8]. Their disk-like shape and flexible cartilaginous pectoral fins give them the ability to travel long distances per stroke, manoeuvre within short spaces, produce strong thrust when leaping out of the water, and reach of a swimming speed up to $2.8 \mathrm{~m} / \mathrm{s}$ at low flapping frequency [4], [6], [9]. This makes them a good candidate to be mimicked as the efficient underwater robotic system.

A number of recent efforts have shown the development of ray inspired robotic prototypes with different bodies, actuations, and swimming characteristics. Each of these works adopted different designs and experimental approaches for underwater locomotion. In [10], a manta ray robot was presented. It has a wing span of $0.6 \mathrm{~m}$ and a swimming speed of $0.7 \mathrm{~m} / \mathrm{s}$. The body is mainly rigid with $3.4 \mathrm{~kg}$ of mass. Later, this robot has been developed with similar weight and wing span but thinner fins [11]. It has a flexible silicone membrane connected along the fin and one degree of freedom for the leading edge. 
It demonstrated a swimming speed of $0.32 \mathrm{~m} / \mathrm{s}$. A shape-memory-alloy (SMA) actuated the micro manta ray has been developed in [12]. It has two SMA wires embedded within the flexible skin. It has a total mass of $354 \mathrm{~g}$ and a wing span of $0.22 \mathrm{~m}$. It is able to demonstrate a swimming speed of $0.057 \mathrm{~m} / \mathrm{s}$. In [13], an ionic-polymer-metal-composite (IMPC) actuated ray robot was reported.

It has a mass of $55 \mathrm{~g}$ and length of $0.11 \mathrm{~m}$. By controlling the fin twisting angle, the power consumption was measured. It demonstrated a swimming speed of $0.0007 \mathrm{~m} / \mathrm{s}$. RoMan-II [14] and RoMan-III [15] are two ray inspired robots designed for underwater locomotion. Their bodies have total mass of $7.3 \mathrm{~kg}$ and $5 \mathrm{~kg}$, respectively. RoMan-II has a body length of $1 \mathrm{~m}$ and swimming speed of $0.4 \mathrm{~m} / \mathrm{s}$, whereas RoMan-III has a body length of $0.88 \mathrm{~m}$ and swimming speed of $0.3 \mathrm{~m} / \mathrm{s}$.

A bionic fin ray robot presented in [5] uses servo motors to actuate the leading edge. It has a weight of $0.76 \mathrm{~kg}$ and a wing span of $0.58 \mathrm{~m}$. It shows a swimming speed of $0.49 \mathrm{~m} / \mathrm{s}$. In [16], two identical soft elastomeric stingray-like robots are developed. Both robots are made of different polymer composites. They are actuated by a single actuator and contain three buoyancy tanks. Each has a mass of $0.486 \mathrm{~kg}$, length of $0.225 \mathrm{~m}$, and swimming speed of $0.08 \mathrm{~m} / \mathrm{s}$.

Another ray inspired soft robot is reported in [17]. It is actuated by pneumatic muscles. It was tested in a static environment (fixed position) in order to examine hydrodynamics. It has a swimming speed of $0.2 \mathrm{~m} / \mathrm{s}$ with a wing span of $0.22 \mathrm{~m}$. In [18], a micro level soft tissue engineered robotic ray was developed. Its real muscle layer is excited by optical excitation. It has a diameter of $0.01 \mathrm{~m}$ and a swimming speed of $0.001 \mathrm{~m} / \mathrm{s}$. A ray inspired propulsor was presented in [19] with an ability to perform undulatory motion using its pectoral fins. It is made of soft polymer and actuated by SMA. With a body length of $0.125 \mathrm{~m}$, it was able to swim with the speed of $0.045 \mathrm{~m} / \mathrm{s}$.

All these works employ various actuation and design methods. There is also a noticeable difference in robot size, actuator type, and overall performance. Most of these robots are in the development stage; some are bulky while others are lightweight but swim very slow. Bulky robots need high torque actuators which result in high power consumption and cost. Similarly, the use of smart actuators such as SMA gives the soft robots flexibility and lightweight bodies but result in high power losses and limited swimming performance.

Beside the issue of actuators, recent advancements in soft robotics [20]-[22] indicate promising possibility to develop more compliant ray-like robots.
Accordingly, a new design strategy focusing on lightweight and compliant robot body may improve overall performance of a ray inspired soft robot.

In this paper, a soft lightweight ray inspired robot is presented. It has an ability to perform underwater propulsion using pectoral fins. It is designed to reduce the actuator power consumption.

In addition, the design strategy indicates a novel multi-purpose of the symmetric pectoral fins. The multi-purpose fins are designed to cater locomotion, go-to-goal, and a lightweight object manipulation. Each fin is actuated by a micro-servo motor which is connected to a cylindrical tendon. The cylindrical tendon is embedded within the elastomeric robot body. An offboard power and control circuit actuate the servo motors according to predefined gait motion. The ray robot is tested in controlled environments both outside and under water. By tuning fin flapping at 2 different flapping amplitudes and 3 different frequencies, the pectoral fin motion is analysed. In addition, the power consumption is also investigated to determine the efficient swimming gait. The soft robot demonstrates two ray-motion mimicking patterns. Moreover, an underwater go-togoal behaviour is also described. This behaviour could be tested in a dynamic experimental setup as a part of the future work.

In the next section, the design of the soft robot is presented by providing a background on the selective morphological features and motion patterns observed in the real rays. Later, the design and fabrication of the soft robot are explained in detail. The experimental setup to perform different performance characterization tests is also presented. In section 3 , the results of pectoral fin testing are reported. In section 4, the testing results are discussed by analysing different parameters to characterize the motion and overall performance of the soft robot. This also identifies the future work. Finally, section 5 summarizes the work.

\section{Materials and Methods \\ 2.1. Design of the Ray Inspired Soft Robot 2.1.1 Background}

Manta rays and stingray have wide disk-like bodies. Figure 1 shows a stingray (a) and manta ray (b) as well as their bone structures. Their fins are made of tiny cartilage bones that are connected to main body muscles in radial direction [7]. By actuating the main muscle that connects the body to the pectoral fin, the thrust is generated across the pectoral fin, resulting in the pectoral motion. 


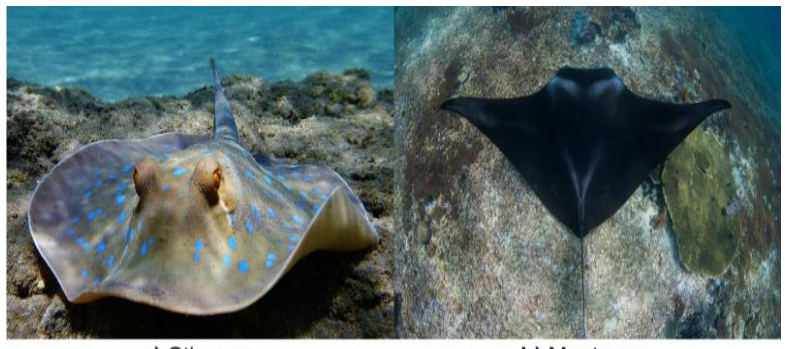

a) Stingray

b) Manta ray

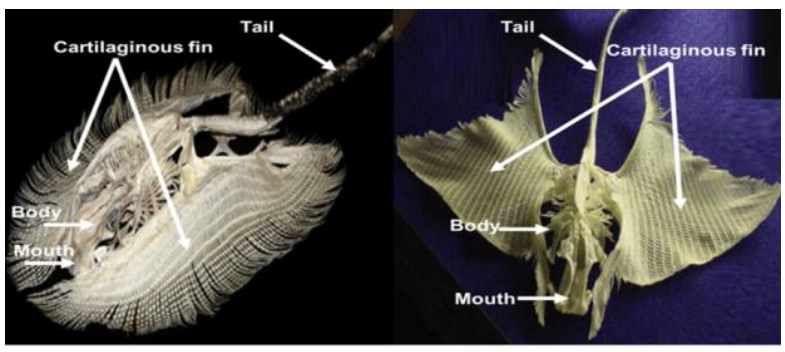

c) Stingray bone structure

d) Manta ray bone structure

Figure 1: A stingray and manta ray: a) A blue spotted stingray using its disk-like body and pectoral fins to calmly manoeuvre underwater (photograph courtesy: W.V. Goethem); b) an oceanic manta ray using its wide flapping pectoral fins performs underwater locomotion (photograph courtesy: I. Ismail); c) Bone structure of stingray [3]; d) Bone structure of manta ray [3].

Biomechanical studies [7], [23], [24] show that the ray cartilage structure and optimal swimming gait contribute to overall swimming performance. In terms of motion, the pectoral fin motion of rays is classified into two types: undulatory and oscillatory motion. This classification depends on the ratio of wavelength $(\lambda)$ of the backward travelling wave to the body length (L) [6]. For manta rays, this wave ratio number of $\lambda / L$ is greater than 1 which is the oscillatory motion. Whereas, stingray have $\lambda / L$ less than 1 which produces undulatory motion. Stingrays (undulators) send traveling waves across the fins using their round shaped pectoral fins, while manta rays (oscillators) use their triangular fins to perform up-and-down flapping [24]. These motion differences have their own advantages. That is, manta rays have excellent thrust and swimming speed which result in high swimming efficiency. On the other hand, stingrays perform undulation with low speed yet highly manoeuvrable underwater locomotion.

By understanding these fundamental body and motion specifications, the soft robot design combines selective features of manta rays and stingrays. It is mainly based on the manta ray because of a desire to obtain good swimming speed, while the thin pectoral edge of the robot is inspired from stingray's morphology in order to have manoeuvrability by undulation behaviour.

\subsubsection{Design}

The ray inspired soft robot design, as shown in Figure 2, is based on the concepts that overall body and pectoral fin dimensions of the robot must suit to execute locomotion behaviors e.g. undulatory and oscillatory motions using the multi-purpose pectoral fins. An earlier work presenting software-based design optimization of this design concept could be seen in [29]. In addition, it has to be lightweight in order to reduce body-load on the actuators. The presented soft robot has three fins arranged in a radial symmetry. The third fin is for controlling direction of motion such that if it tilts to a side, the thrust direction of the other two fins will follow the profile of the body and tilted third fin, resulting in changing of the robot moving direction. This idea is further illustrated in Figure 3. It shows that each fin can steer the robot in a particular direction. This provides possibility to make the soft robot navigating in multiple directions with minor control signal tuning. By letting the two fins perform synchronized flapping, the third fin acts as a standalone direction or inclination controller.

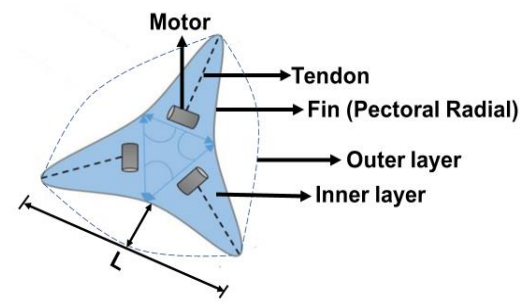

Figure 2: Design concept schematic of the ray inspired soft robot showing three symmetric fins. The fin motion is actuated by a tendon that is connected to a servo motor.

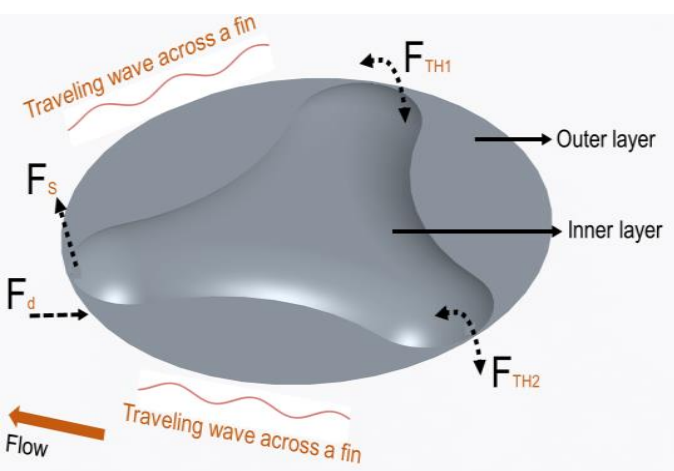

Figure 3: A typical motion pattern resulting in forward or directional motion of the robot when two fins flap synchronously while the third fin controls the body direction or inclination. Inner layer generates

the flapping thrust while the outer layer helps in maneuverability. Here, CAD rendering shows the soft robot positioned to swim in a direction when $F_{d}$ is the hydrodynamic drag, $F_{T H 1}$ and $F_{T H 2}$ are side fin flapping thrusts. $F_{s}$ is the minor force thrust generated by the motion of steering fin. 
Table 1 lists specifications of the soft robot. The robot main body has two layers in different thickness. The inner layer is composed of three symmetric fins. It has a thickness of $3 \mathrm{~mm}$ and a sideto side length of $12.5 \mathrm{~cm}$. The outer layer has a thickness of $1 \mathrm{~mm}$ and variable length from 1 to $2 \mathrm{~cm}$ on each side.

Table 1. Specifications of the soft robot

\begin{tabular}{|c|c|}
\hline Parameter & Value \\
\hline $\begin{array}{c}\text { Main body length (side to } \\
\text { side) }\end{array}$ & $12.5 \mathrm{~cm}$ \\
\hline Main body thickness & $3 \mathrm{~mm}$ \\
\hline $\begin{array}{c}\text { Layer 1: fin chord width } \\
\text { (symmetric) }\end{array}$ & $6.5 \mathrm{~cm}$ \\
\hline Length of extended layer 2 & $1-2 \mathrm{~cm}$ \\
\hline Thickness of extended layer 2 & $1 \mathrm{~mm}$ \\
\hline Length of cylindrical tendon & $5 \mathrm{~cm}$ \\
\hline $\begin{array}{c}\text { Diameter of cylindrical } \\
\text { tendon }\end{array}$ & $5 \mathrm{~mm}$ \\
\hline Main body mass & $36 \mathrm{~g}$ \\
\hline Actuation type & Electrical \\
\hline Controller & Uno R3 \\
\hline Actuator & servo (SG90) \\
\hline Basic degrees of freedom & 3 \\
\hline
\end{tabular}

For actuation, the micro servo motor (Tower Pro SG90) is chosen to actuate the soft robot. This is to leverage on its small size and fair efficiency to support fin flapping. Three of the servo motors are implanted into the elastomeric body. They act as the main force generation mechanism similar to the main muscle of the ray body which initiates the motion towards the edge of a cartilaginous pectoral fin. To distribute and transfer motion from a servo motor to the pectoral fin, a cylindrical thermo-plastic tendon is used. An Arduino Uno board was used to control the servo motors which is powered through a $9 \mathrm{~V} \mathrm{AC}$-to-DC power adaptor. Currently, the power supply and controller are kept out of the robot body in order to reduce body load while focusing on fin behavior.

\subsection{Fabrication Process}

A complete step-by-step process to fabricate the ray inspired soft robot is illustrated in Figure 4. First, a two-side square shaped $(16 \mathrm{~cm} \times 16 \mathrm{~cm})$ mold was designed by the Creo Parametric (PTC) software. Then, a CNC machine with $0.05 \mathrm{~mm}$ precision was used to prepare the top and bottom sides of the mold. The bottom cavity mold was machined from an acrylic sheet of $1.5 \mathrm{~cm}$ thickness. The top mold was machined from an acyclic sheet of $1 \mathrm{~cm}$ thickness. To make the soft robot body, RTV-225 silicone elastomer (GGC, Taiwan) was used. The RTV-225 raw material comes in part 'a' and part 'b'. Part 'a' is the silicone elastomer, and part ' $b$ ' is the hardener agent. Using a mixing ratio of $96: 4$ (\% by weight) of part 'a' to part 'b', a mixture was prepared. By pouring it into the bottom cavity mold, the top mold was pressed and tightened together. After 2 hours of curing at room temperature, the soft elastomeric body was prepared. Later, a thermo-plastic cylindrical tendon was placed inside the inner layer of each fin. Then, the additional RTV-225 mixture was filled to cover the tendons. This resulted in joining the tendons with the soft body after two more hours of curing. For each fin, the micro servo motor was wrapped in a thin elastic rubber layer and placed at the center of the robot body, next to the tendon. The motor horn was attached with the tendon in order to force it to bend. The motors were fixed with the soft body by using thin plastic straps.

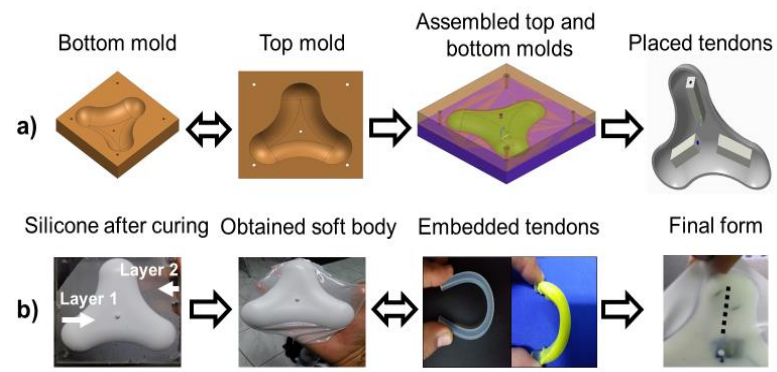

Figure 4: An overview of the soft robot fabrication process: a) The $3 D$ design of the mold and resultant shape of the soft robot; b) The RTV-225 was poured into the bottom cavity mold. By joining top and bottom sides, the soft robot body was prepared. Later on, the flexible cylindrical tendons were placed and filled with additional RTV-225 (dotted line in final form indicates position of tendon). The servo motor was attached to the tendon for testing of the soft pectoral fin.

\subsection{Control of the Locomotion Patterns}

Typically, flapping of the fin involves controlling a vertically oscillating sinusoidal wave. This can be written as:

$\mathrm{x}(\mathrm{t})=\mathrm{A} \sin (2 \pi \mathrm{ft})$,

where $x(t)$ is the continuous signal that oscillates the fins. The control variables are the flapping frequency (f) and flapping amplitude (A). In this research, 6 combinations of three frequencies $(0.5 \mathrm{~Hz}, 1 \mathrm{~Hz}$, and $2 \mathrm{~Hz})$ and two amplitudes $\left(30^{\circ}\right.$ and $\left.45^{\circ}\right)$ are investigated.

The control can be achieved by tuning the pulse width modulation (PWM) of the servo motor to accommodate these combinations. In broader sense, oscillation and undulation is generated with various gait control parameters' tuning such that the global motion can be adjusted with coupling of local actuators. Following this, Different swimming gaits were mapped. Figure 5 (a) shows upstroke and downstroke definitions for a cycle of flapping which originates from the neutral point. An efficient gait was characterized based on power consumption of 
the actuator and traveling displacement of the fin from a marked position, as it glided by one flapping cycle. This was performed with an open-loop/feedforward control process as shown in Figure 5 (b).

a)

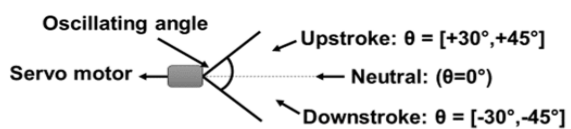

b)

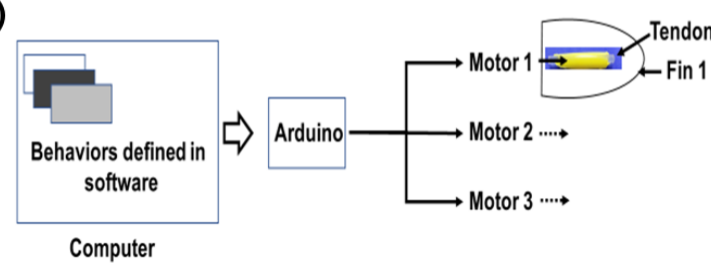

Figure 5: Control scheme overview. a) Illustration of the servo flapping by position control as it vertically oscillates resulting in upstroke and downstroke motion of the fin. b) An overview of the open-loop flapping control process.

\subsection{Experimental Setup for the Fin Testing}

The soft robot fins were tested in two scenarios. First, the test was performed outside water, and later underwater with different flapping amplitudes and frequencies. The experiment was setup to observe the motion of the fins (See Figure 6). A fixed hinge was used to hold the soft body of the robot.

The servo motor was fixed with the hinge and its horn was attached with the tendon to flap the fin vertically. An Arduino Uno board controlled the input signal to the servo motor. For the underwater experiment, a small water tank was used. During the underwater testing, the hinge was initially fixed and later adjustable to vary the depth level. This was to observe possible dynamics effect of the free surface on the fin motion or the force generated by the flapping motion which acts against the weight of the body during the fin motion.

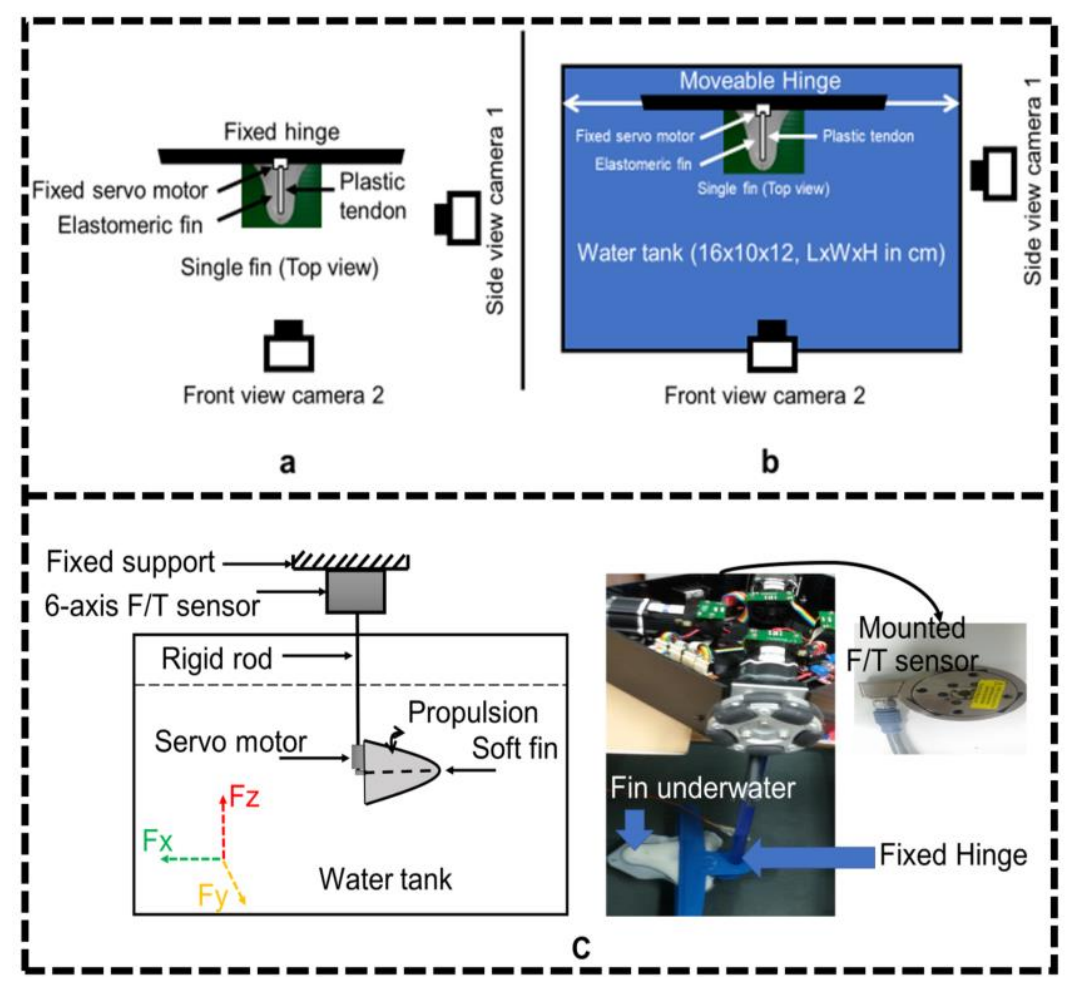

Figure 6: An overview of the experimental setups: a) The soft pectoral fin was attached to a fixed hinge and the test was performed outside water; b) For the underwater testing, the soft pectoral fin was immersed in a water tank for 4-8 cm depth. Two cameras were used to record pectoral fin motion in both scenarios; c) Experimental setup to test a fin thrust in a direction underwater.

In order to measure the material-provided tendon reaction force exerted by the actuator during the flapping, the experimental setup was modified. The hook of a weight scale was attached with a fin the center of it. For maximum amplitude of flapping, the reaction force of the tendon was measured during outside and underwater fin flapping. To test a fin thrust, experimental setup (Figure 6c) included fin fixed with a force/torque sensor (SI-130-10, ATI,
USA). For the power consumption measurement, a digital oscilloscope (Instek GDS-806S, Taiwan) was used to analyze the current drawn by the actuator for different flapping gaits. A variable resistor was adjusted to be $5.5 \mathrm{ohm}$. It was connected to the positive voltage wire of the servo motor, allowing measurement of the current variation. The recorded current was later calculated for estimated power consumption of the actuator. In order to examine the 
motion of pectoral fins, two cameras (G2-G7102, Samsung) were used for viewing two sides of the flapping pectoral fin. Camera 1 recorded the oscillatory motion of the fin from the side view. Camera 2 was placed on the front view such that it could record possible undulatory behavior of the thin pectoral fin edges. The recorded motion was later analyzed frame-by-frame to understand the behavior of pectoral fin motion.

\section{Results}

First it is helpful to see Figure 7 which shows a set of frequency and amplitude scenarios of the fin flapping. By performing these gait variations, motion of the pectoral fin was analyzed through recordings of the pectoral fin motion.

Figure 8 shows a set of photographs taken at run time when the soft fin completed a flapping cycle. The motor rotations of $60^{\circ}$ and $90^{\circ}$ create the flapping amplitude of $30^{\circ}$ and $45^{\circ}$, respectively. Based on the video analysis of camera 2, the motion of the fin shows minor rearward traveling wave. Figure 8 presents the oscillatory fin motion. At the same time, Figure 9 shows minor undulatory behavior observed by the front view on camera 2 .
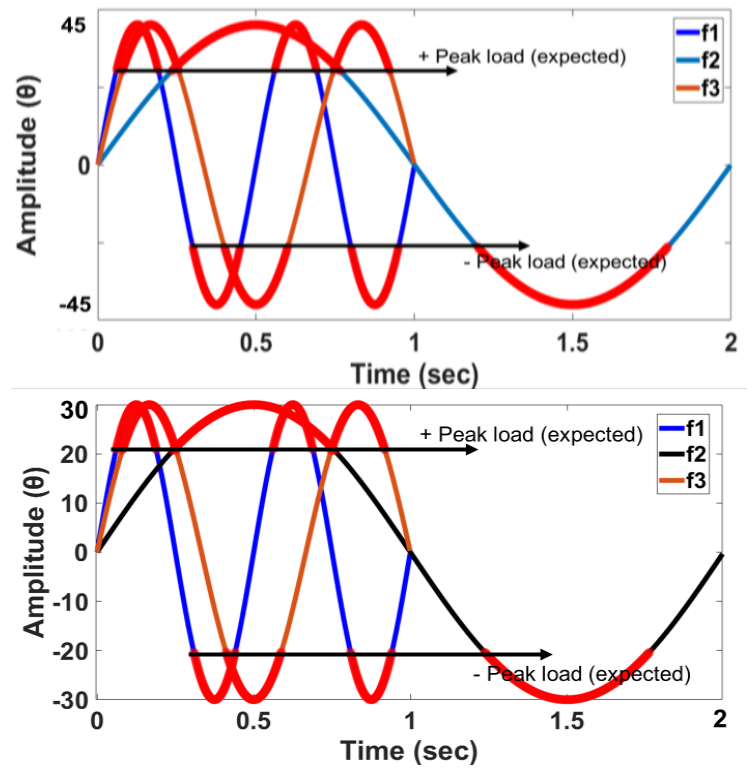

Figure 7: An overview of different fin testing control inputs in terms of peak-to-peak amplitude (Top: 60', Bottom: $\left.90^{\circ}\right)$ and frequency scenarios $(f 1, f 2$, and $f 3$ are three tested frequencies). The peak load indicates parts of the control signals that the actuator may experience most power consumption during upstroke and down-stroke of flapping.

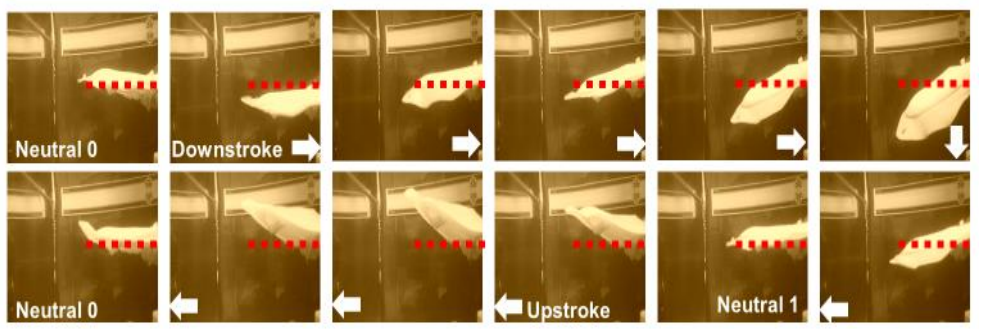

Figure 8: The motion of the soft robotic pectoral fin in one cycle of flapping underwater. By testing on different frequency and amplitude scenarios, the fin preformed an oscillatory motion across the pectoral fins. The downstroke motion frames are shown at 40ms interval, whereas the upstroke motion frames are shown at 83ms interval. The efficient flapping gait was observed at $f=2 \mathrm{~Hz}$, and $A=30^{\circ}$ which was recorded by the side view camera 1. The red dotted line shows the neutral axis, which separate the upstroke and downstroke flapping.
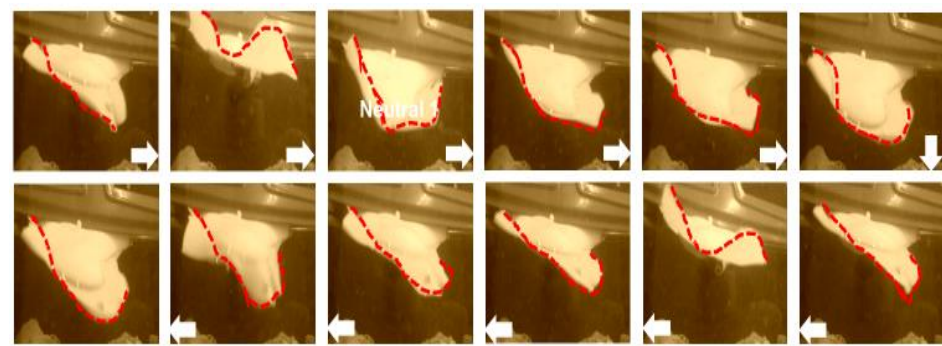

Figure 9: The motion of the soft pectoral fin flapping underwater. The dash line indicates variation of travelling wave across the pectoral fin. By observing the front view camera 2, a minor undulatory motion was found.

Further classifying the fin motion, it was found that at $\mathrm{f}=2 \mathrm{~Hz}$ and $\mathrm{A}=30^{\circ}$, the soft robot demonstrated the better underwater fin propulsion than the other flapping combination. This efficient gait identification was evaluated by the generated thrust and tendon reaction force as it flapped underwater (see Figure 10).

Based on the traveling distance on one flapping cycle of different gaits, the robot velocities were calculated (see Figure 11). 
To further characterize the efficient gait, the current drawn by the actuator was measured, and converted to an estimated power consumption (See Figure 12 and Figure 13)

\section{Discussion}

The presented soft robot design has demonstrated complete oscillatory and minor undulatory motion behaviour across the pectoral fins. Use of the third fin could provide versatility to navigate but would make the control complicated. Length of the robot, dimension and stiffness of the fin, and overall light body weight allow the robot to perform underwater locomotion similar to other robots [4], [5], [8], [10][13], [15], [17], [19].

During the fin testing, the following critical observations were made:
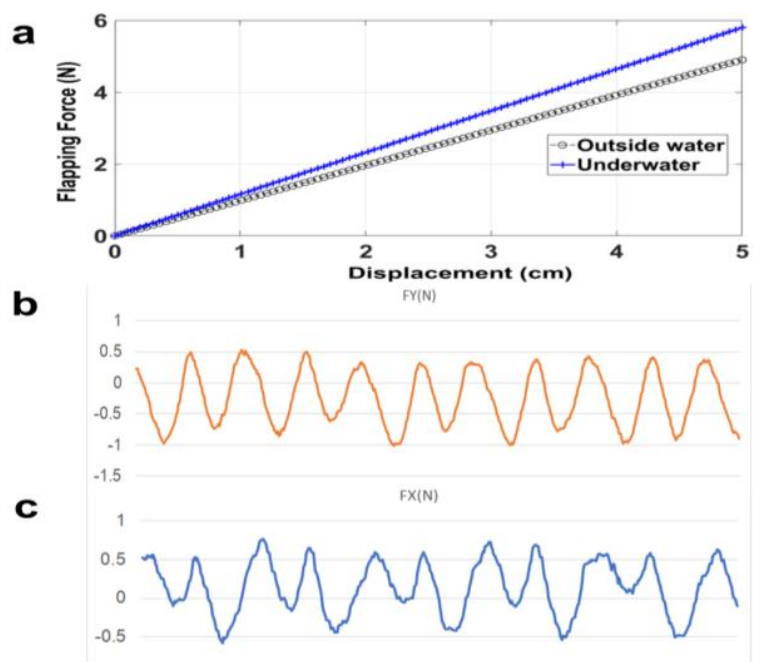

Figure 10: a) The relationship between the peak-to-peak vertical fin tip displacement and the flapping (reaction) force of the tendon that was tested outside and under water. b) Real time Y-direction varying fin-thrust recorded underwater using force sensor. c) Real time X-direction varying fin-thrust underwater.

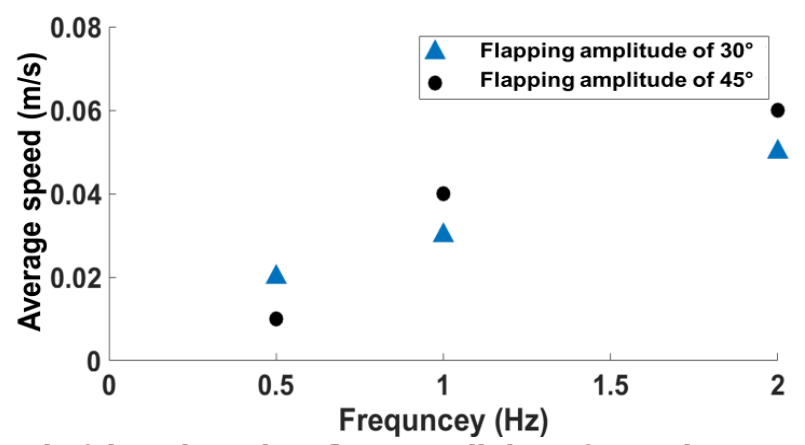

Figure 11: The estimated speed of the robot when flapping all three fins underwater with different amplitudes. This estimation is based on the measured distance of the fin for one complete flapping stroke. Multiple measurements have been done using frame-by-frame video analysis of the marked body point trajectory. Then, the speed was estimated according to frequency and amplitude of flapping.

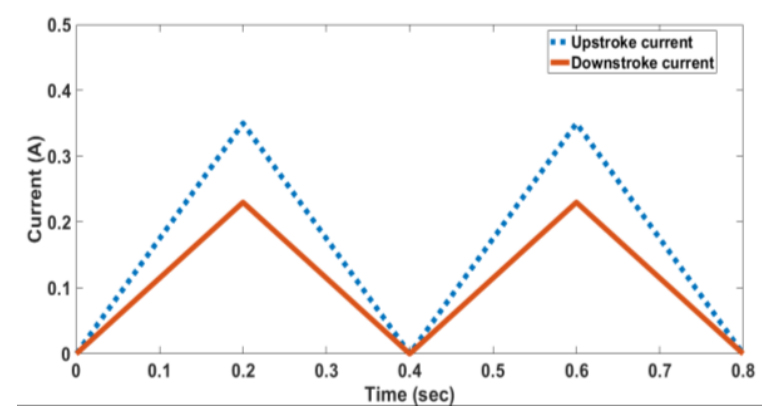

Figure 12. Variation of the current (I) drawn by the actuator during a full flapping stroke.

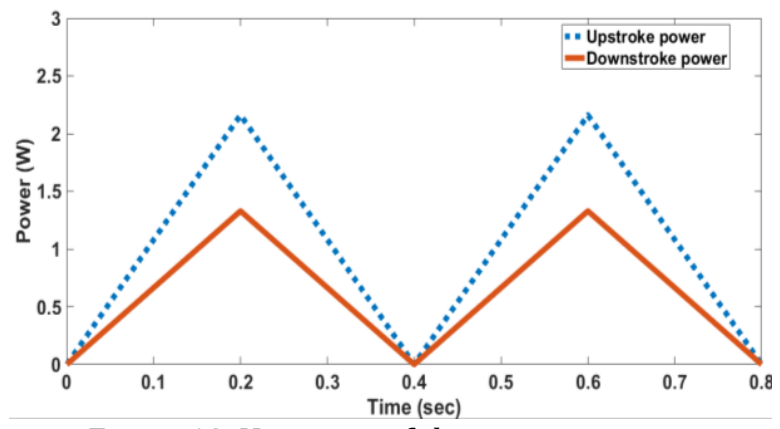

Figure 13. Variation of the actuator power consumption (P) during a full flapping stroke. 
- After multiple iterations of fin testing, a tradeoff in the fin propulsive performance was noted for different gaits. The outside and underwater testing revealed that at the frequency of $2 \mathrm{~Hz}$ and $30^{\circ}$ flapping amplitude, the robot fin was able to demonstrate a complete oscillatory and minor undulatory motion with sufficient level of thrust. At the frequency of $0.5 \mathrm{~Hz}$ and flapping amplitude of $45^{\circ}$, the pectoral flapping was the slowest but minor undulatory motion was obviously observed.

In other cases, the pectoral fin behaviour was at the intermediate level. In terms of upstroke and downstroke displacement away from the neutral position, it was observed that peak-to-peak displacement for a cycle of flapping was $5 \mathrm{~cm}$ for the flapping amplitude of $30^{\circ}$, and $6 \mathrm{~cm}$ for the flapping amplitude of $45^{\circ}$. This minor difference in flapping displacements, sufficient thrust, and ability to perform continuous oscillatory behaviour guide that $\mathrm{f}=2 \mathrm{~Hz}$ and $\mathrm{A}=30^{\circ}$ are the optimal gait parameters. However, this could be further optimized to accommodate more dynamic nature of swimming behaviours.

- The outer thin layer of the fin supported and exhibited the undulatory behaviour only during the underwater testing. The peak of the travelling sinusoidal wave on the pectoral fin edges was controlled by the tendon. The leftover thin layer allowed the wave to travel from one edge to another edge of the pectoral fin. However, there were many non-uniform undulatory waves that needed to be further studied and characterized in order to develop more predictable undulatory behaviour.

Additionally, other material types such as a thermo-plastic elastomer [30] could be investigated to fabricate inner layer or the tendon while keeping the outer layer to be made of silicone.

This will be helpful to explore other material options for fabrication as well as their impact on the embodied behaviour.

- For all gaits, reaction force measurements of the fin show that the force is higher under water than outside water. It was because of the inertial fluid effects (added mass) that contribute to it due to difference in density and viscosity of the tested mediums. Also, it is visible that for the fin testing underwater, $x-y$ thrust is low for a directional motion. Here, $\mathrm{x}$ direction means right/left body thrust, whereas $y$ direction is front/back body thrust. Its tuning and further multi-axis (e.g. by including $\mathrm{x}$-direction up/down thrust) thrust characterization to accommodate a directional motion is part of future development which will focus control of swimming directions. Also, body symmetry and resultant mutual thrusts affecting on the motion are crucial elements and need to be investigated to obtain useful correlation for further design optimization.

- In terms of power consumption, the measured current (I) of all gaits suggest that the motor draws more current during the upstroke flapping than that of the downstroke flapping. Besides the higher power resistance during upstroke, this is due to the position of the motor/tendon inside the inner layer of silicone.

In addition to the single fin testing in a controlled environment, the robot movement could be classified as follows.

- Two fin flapping when the third fin controls traveling direction.

- All three fins flap to perform a specific manoeuvre.

- Two fins grasp a lightweight object while the third fin flaps to move the object to a desired location.

Figure 14 illustrates an overview of possible locomotion patterns. The assumption is such that the soft robot is equipped with necessary sensors and actuators. In case that the robot needs to move from point $A$ to point $A^{\prime}, B$, or $B^{\prime}$, with the presented body orientation, it can oscillate the two fins to produce forward thrust and adjust the third fin to navigate to $B^{\prime}$. However, for other points, the robot body needs to re-orient itself. The three-fin symmetric design allows the robot to change the orientation with a specific manoeuvre. This could save some power consumption in comparison to completely steer the body toward a target point.

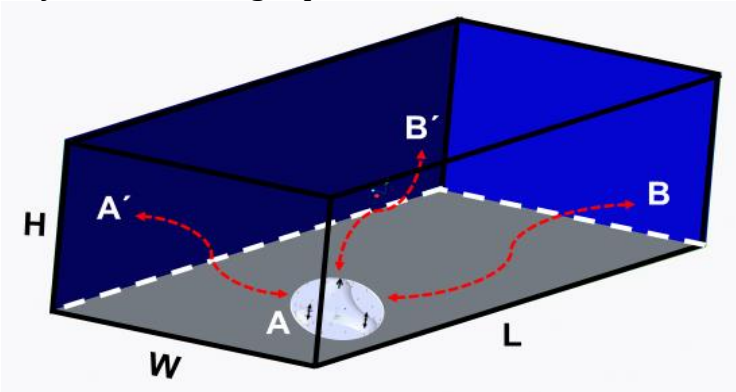

Figure 14. The overview of a go-to-goal scheme where three-fin symmetric design of the soft robot provides ease manoeuvre toward a goal position. Point $A, A^{\prime}, B$, and $B^{\prime}$ are under water. At start, the robot is at the bottom at $A$, and it needs to navigate to other goal points. This could be done by flapping two of the fins for the forward thrust and the third fin for controlling inclination or direction.

Previous works of ray-like robots have shown that swimming speed depends on the type of actuator and employed gait [4], [5], [8], [10]-[13], [15], [17], [19]. In this work, the servo motors provided basic level of flapping locomotion. However, the actuator flapping gait would be more complicated as the robot movement become more complex. Currently, the soft robot fin testing has been performed at the underwater depth of 4 to $8 \mathrm{~cm}$ in the controlled environment. For scenarios such as a go-to-goal behaviour with more underwater depth and continuously changing flapping gait, the actuator would need to be updated. Additionally, the soft 
robot has not been tested in a dynamic environment. More studies are required on the optimized level of fin stiffness and integration of supportive control components such as a buoyancy control unit in order to develop a body stabilizing control scheme.

Moreover, to perform dynamic gait manoeuvres such as a go-to-go al navigation as illustrated in Figure 14, the soft robot needs to be equipped with necessary sensors to perceive the environment. Further modelling and experimental approaches [25]-[28] could be adopted to understand hydrodynamics related to the ray robot motion with the real ray. This would help to characterize performance of the presented soft robot.

\section{Conclusion}

In this paper, a new design of the ray inspired soft robot is presented. The soft robot is designed such that it could show undulatory and oscillatory motions across the pectoral fins. Using acrylic mold, RTV-225 silicone elastomer was used to fabricate the soft body of the robot. The soft robot has three symmetric pectoral fins, each driven by a servo motor. The robot body composes of two different thickness layers. The body has an extended thin layer which allows to observe the undulatory behavior across the pectoral fins. Cylindrical thermoplastic tendons were embedded within the elastomeric body of the robot to transfer the motion from the servo motor to the pectoral fin. This approach of using a tendon to control the fin with the proposed flapping yields real ray-like pectoral motion. The overall efficient gait was observed at flapping frequency of $2 \mathrm{~Hz}$ and amplitude of $30^{\circ}$. This was noted by adjusting the fin flapping at different amplitudes and frequencies through a controller. The robot motion was further analyzed using video recordings of the pectoral fin motion. The results have shown that the robot fin can perform major oscillatory and minor undulatory motions. A simple go-to-goal scheme was suggested as a part of future work. Further work would develop this new design of the ray inspired soft robot as a functional underwater robotic platform.

\section{Acknowledgement}

Authors are thankful to Mr. Peerayut Saekow, Dr. Paramin Neranon, Mr. Amir Souhail, Mr. Prakarn Jaroonsorn, and Ms. Kamonrat Tangudomkit for supporting in different stages of this work. Our appreciation goes to Ms. Wendy van Goethem and Mr. Ismadi Ismail for providing photographs of stingray and manta ray (Figure $1 ; \mathrm{a}, \mathrm{b}$ ), respectively. We are also grateful to all anonymous reviewers for their constructive feedback. This work was funded by the Higher Education Research Promotion of the Higher Education Commission and the Education
Hub Program for the Southern Region of ASEAN countries. We also acknowledge the support of the department of mechanical engineering and faculty of engineering, Prince of Songkla University for provision of resources to carry out this research.

\section{References}

[1] F. Delcomyn, "Biologically Inspired Robots," Bioinspiration Robot. Walk. Climbing Robots, 2007.

[2] S. Zimmerman and A. Abdelkefi, "Review of marine animals and bioinspired robotic vehicles: Classifications and characteristics," Prog. Aerosp. Sci., vol. 93, pp. 95-119, Aug. 2017.

[3] R. Salazar, V. Fuentes, and A. Abdelkefi, "Classification of biological and bioinspired aquatic systems: A review," Ocean Eng., vol. 148, pp. 75-114, Jan. 2018.

[4] K. W. Moored, F. E. Fish, T. H. Kemp, and H. BartSmith, "Batoid Fishes: Inspiration for the Next Generation of Underwater Robots," Mar. Technol. Soc. J., vol. 45, no. 4, pp. 99-109, Jul. 2011.

[5] C. M. Chew, Q. Y. Lim, and K. S. Yeo, "Development of propulsion mechanism for Robot Manta Ray," in 2015 IEEE International Conference on Robotics and Biomimetics (ROBIO), 2015, pp. 1918-1923.

[6] L. J. Rosenberger, "Pectoral fin locomotion in batoid fishes: undulation versus oscillation," J. Exp. Biol., vol. 204, no. 2, pp. 379-394, Jan. 2001.

[7] J. T. Schaefer and A. P. Summers, "Batoid wing skeletal structure: Novel morphologies, mechanical implications, and phylogenetic patterns," J. Morphol., vol. 264, no. 3, pp. 298313, Jun. 2005.

[8] P. V. y Alvarado, "Hydrodynamic performance of a soft body under-actuated batoid robot," in 2011 IEEE International Conference on Robotics and Biomimetics, 2011, pp. 1712-1717.

[9] A. J. Smits, K. W. Moored, and P. A. Dewey, "The Swimming of Manta Rays," in Fluid-StructureSound Interactions and Control, Springer, Berlin, Heidelberg, 2014, pp. 291-300.

[10] J. Gao, S. Bi, Y. Xu, and C. Liu, "Development and design of a robotic manta ray featuring flexible pectoral fins," Proc. IEEE Int. Conf. Robot. Biomim., vol. 2007, pp. 519-523, 2007.

[11] C. Niu, L. Zhang, S. Bi, and Y. Cai, "Development and depth control of a robotic fish mimicking cownose ray," in 2012 IEEE International Conference on Robotics and Biomimetics (ROBIO), 2012, pp. 814-818.

[12] Z. Wang, Y. Wang, J. Li, and G. Hang, "A micro biomimetic manta ray robot fish actuated by SMA," in 2009 IEEE International Conference on Robotics and Biomimetics (ROBIO), 2009, pp. 1809-1813. 
[13] Z. Chen, T. I. Um, and H. Bart-Smith, "Bioinspired robotic manta ray powered by ionic polymer-metal composite artificial muscles," Int. J. Smart Nano Mater., vol. 3, no. 4, pp. 296-308, Dec. 2012.

[14] C. Zhou and K. H. Low, "Design and Locomotion Control of a Biomimetic Underwater Vehicle with Fin Propulsion," IEEEASME Trans. Mechatron., vol. 17, no. 1, pp. 25-35, Feb. 2012.

[15] K. H. Low, C. Zhou, G. Seet, S. Bi, and Y. Cai, "Improvement and testing of a robotic manta ray (RoMan-III)," in 2011 IEEE International Conference on Robotics and Biomimetics, 2011, pp. 1730-1735.

[16] P. V. y Alvarado, S. Chin, W. Larson, A. Mazumdar, and K. Youcef-Toumi, "A soft body under-actuated approach to multi degree of freedom biomimetic robots: A stingray example," in 2010 3rd IEEE RAS EMBS International Conference on Biomedical Robotics and Biomechatronics, 2010, pp. 473478.

[17] Y. Cai, S. Bi, L. Zhang, and J. Gao, "Design of a robotic fish propelled by oscillating flexible pectoral foils," in 2009 IEEE/RSJ International Conference on Intelligent Robots and Systems, 2009, pp. 2138-2142.

[18] S.-J. Park et al., "Phototactic guidance of a tissue-engineered soft-robotic ray," Science, vol. 353, no. 6295, pp. 158-162, Jul. 2016.

[19] H.-S. Kim, J.-Y. Lee, W.-S. Chu, and S.-H. Ahn, "Design and Fabrication of Soft Morphing Ray Propulsor: Undulator and Oscillator," Soft Robot., vol. 4, no. 1, pp. 49-60, Dec. 2016.

[20] S. Kim, C. Laschi, and B. Trimmer, "Soft robotics: a bioinspired evolution in robotics," Trends Biotechnol., vol. 31, no. 5, pp. 287-294, May 2013.

[21] C. Majidi, "Soft Robotics: A PerspectiveCurrent Trends and Prospects for the Future," Soft Robot., vol. 1, no. 1, pp. 5-11, Jul. 2013.

[22] D. Trivedi, C. D. Rahn, W. M. Kier, and I. D. Walker, "Soft Robotics: Biological Inspiration, State of the Art, and Future Research," Applied Bionics and Biomechanics, 2008. [Online]. Available: https://www.hindawi.com/journals/abb/2008/ 520417/abs/.
[23] R. S. Russo, S. S. Blemker, F. E. Fish, and H. Bart-Smith, "Biomechanical model of batoid (skates and rays) pectoral fins predicts the influence of skeletal structure on fin kinematics: implications for bio-inspired design," Bioinspir. Biomim., vol. 10, no. 4, p. 046002, 2015.

[24] X. Liu, F. Fish, R. S. Russo, S. S. Blemker, and T. Iwasaki, "Modeling and Optimality Analysis of Pectoral Fin Locomotion," in Neuromechanical Modeling of Posture and Locomotion, Springer, New York, NY, 2016, pp. 309-332.

[25] W. Chen, Z. Wu, J. Liu, S. Shi, and Y. Zhou, "Numerical simulation of batoid locomotion," J. Hydrodyn. Ser B, vol. 23, no. 5, pp. 594-600, Oct. 2011.

[26] R. G. B. Ii, I. Borazjani, E. L. Blevins, and G. V. Lauder, "Hydrodynamics of swimming in stingrays: numerical simulations and the role of the leading-edge vortex," J. Fluid Mech., vol. 788, pp. 407-443, Feb. 2016.

[27] G. Iosilevskii, "Hydrodynamics of the undulatory swimming gait of batoid fishes," Eur. J. Mech. - BFluids, vol. 45, pp. 12-19, May 2014.

[28] L. Wen, T. M. Wang, G. H. Wu, and J. H. Liang, "Hydrodynamic investigation of a self-propelled robotic fish based on a force-feedback control method," Bioinspir. Biomim, vol. 7, no. 3, p. 036012 , May 2012.

[29] M. B. Khan and P. Smithmaitrie, "Design and Improvement of a Manta Inspired Soft Robot Using Finite Element Analysis," in 32nd Conference of Mechanical Engineering Network of Thailand (MeNett), Thailand, 2018.

[30] M. B. Khan, P. Smithmaitrie, and E. Kalkornsurapranee, "A Comparative Study of RTV-225 Silicone Elastomer and Natural Rubber Based Thermo-Elastomer for Soft Robotic Applications," in 7th International Conference on Design and Concurrent Engineering (iDECON), Malaysia, 2018. 